\title{
Multifunktionale Regenwasserbewirtschaftung durch smarte Regentonnen
}

\author{
Martin Oberascher · Jonatan Zischg · Ulrich Kastlunger · Martin Schöpf · Carolina Kinzel · Christoph Zingerle · \\ Wolfgang Rauch · Robert Sitzenfrei
}

Online publiziert: 6. Mai 2019

(C) Der/die Autor(en) 2019

Zusammenfassung Regentonnen sind temporäre Speichereinheiten und werden für eine multifunktionale Regenwasserbewirtschaftung eingesetzt, mit der Aufgabe, Niederschlagswasser für Bewässerungszwecke bereitzustellen. Jedoch weisen einzelne Regentonnen im Vergleich zum zentralen Regenbewirtschaftungssystem kleine Speichereinheiten auf. Um Regentonnen dennoch effizient in das Gesamtsystem einbinden zu können, sind daher neue Ansätze für den Betrieb erforderlich, wie zum Beispiel das Konzept der smarten Regentonnen (SRT). Die SRT sind kleine Speichereinheiten, die in Echtzeit kontrolliert und gesteuert werden. Durch die Einbindung in eine smarte Wasserinfrastruktur sind die aktuellen Systemzustände bekannt, wodurch die SRT hoch effizient genutzt werden können, um (a) die Spitzenabflüsse im Kanalsystem zu reduzieren und (b) den Trinkwasserverbrauch durch die Verwendung von Regenwasser für die Bewässerung zu vermindern. Um die Wirksamkeit des SRTKonzeptes $\mathrm{zu}$ demonstrieren, wurde in Kombination mit der Wettervorhersage ein zweistufiger Ansatz verfolgt: Erstens wurde ein Versuchsmodell der SRT entwickelt, welches im Frühjahr 2019 am Campus Technik der Universi-

DI M. Oberascher, BSc $(\bowtie)$.

DI J. Zischg • U. Kastlunger, BSc .

M. Schöpf, MSc · Dr. C. Kinzel .

Univ.-Prof. DI Dr. W. Rauch .

Univ.-Prof. DI Dr. R. Sitzenfrei

Arbeitsbereich Umwelttechnik,

Institut für Infrastruktur,

Universität Innsbruck,

Technikerstraße 13, 6020 Innsbruck,

Österreich

martin.oberascher@uibk.ac.at

\section{Zingerle, MSc}

Zentralanstalt für Meteorologie

und Geodynamik (ZAMG),

Fürstenweg 180, 6020 Innsbruck,

Österreich tät Innsbruck für Demonstrations- und Testzwecke aufgestellt und betrieben wird. Des Weiteren wurde durch Simulationen eine Wirksamkeitsanalyse auf Haushaltsebene durchgeführt. Wie die Ergebnisse zeigen, kann durch die SRT das Retentionsvolumen im Vergleich $\mathrm{zu}$ einer herkömmlichen Regentonne deutlich erhöht werden. Die SRT bieten neben der Technik (Einbindung in das Gesamtsystem) den Vorteil, dass sie einfach in das bestehende System integriert werden können, und bei einer großflächigen Implementierung steht in Summe ein bedeutendes Speichervolumen zur Verfügung.

Schlüsselwörter Digitalisierung Echtzeitsteuerung · Intelligentes Regenwassermanagement . Regentonnen · Wettervorhersage

\section{Multifunctional rainwater} management through smart rainwater barrels

Abstract Rain barrels are temporary storage volumes and can be used for a multi-functional rainwater management, with the purpose of retaining stormwater for irrigation. However, compared to the overall system rain barrels provide only small storage volumes. Therefore, new possibilities for the operation are needed, such as the smart rain barrel (SRB) concept. The SRBs are micro-scale storage volumes which are monitored and controlled in real-time. Through the integration in a smart water infrastructure the overall state of the system is known and the SRBs can be used efficiently to (a) reduce peak run-off rates in the sewer system and (b) decrease the potable water consumption by using rain water for irrigation. To show the effectiveness of the SRB concept, a two-stage approach in combination with weather forecasts was used. First, a test model was developed, which is situated at the Campus Technik of the University of Innsbruck and will be implemented for demonstration and test purposes in spring 2019. Second, simulations on household level were carried out. As the results show, the retention volume can be increased significantly through the SRBs by adding weather forecasts to the control strategy. Besides the technology (integrating the SRBs into the main system), the SRBs offer the advantage that they can be easily integrated into existing systems and, in case of largescale implementation, a significant total retention volume is available.

Keywords Digitalisation · Intelligent Stormwater management · Rain barrels · Real-time control · Weather forecast

\section{Einleitung}

Urbanisierung und Klimawandel sowie die Erhaltung sind gegenwärtige Hauptherausforderungen für die urbane Wasserinfrastruktur und werden auch in der Zukunft von hoher Relevanz sein. Einerseits wird durch den Bau von Straßen und Gebäuden der Anteil der versiegelten Fläche erhöht, wodurch der oberflächliche Niederschlagsabfluss zunimmt (Fletcher et al. 2013). Anderseits haben sich durch den Klimawandel Dauer, Intensität und Wiederkehrzeit von Starkregenereignissen verändert (García et al. 2015). Durch das Zusammenwirken von Urbanisierung und Klimawandel hat sich die Wahrscheinlichkeit einer Überlastung der existierenden, urbanen Siedlungsentwässerung erhöht (Yazdanfar and Sharma 2015) sowie die Belastung der natürlichen Wasserressourcen für die Trinkwasserbereitstellung zugenommen (Cominola et al. 2015). Daher wurden in den letzten Jahrzehnten vermehrt dezentrale Anlagenteile zur Lösung lokaler Probleme in urbanen Gebieten eingesetzt, wodurch der Betrieb der urbanen Wasserinfrastruktur 
komplexer und vielfältiger wurde (Fletcher et al. 2015).

Dezentrale Anlagenteile in der Wasserversorgung nutzen beispielsweise Regenwasser für „Nicht-Trinkwasser“Anwendungen wie Bewässerung oder WC-Spülung und reduzieren dadurch den Trinkwasserverbrauch. Dabei wird Niederschlagswasser von Dachflächen gesammelt, zwischengespeichert und bei Bedarf wieder abgegeben (Fewkes and Butler 2000). Durch eine empirische Bewertung der Leistung zeigten Ward et al. (2012), dass die tatsächliche Leistung bei einer Regenwasserbewirtschaftung von der idealen Bemessungsannahme abweicht. Trotz dieser Abweichung lag die Kapitalrückzahlung zwischen 6 und 11 Jahren und es wurde deutlich, wie wichtig eine kontinuierliche Überwachung solcher Anlagen ist. Notaro et al. (2017) versuchten, die Größe einer Regenwasseranlage für die zuverlässige Nutzung für die Bewässerung sowie für WC-Spülungen in einem mediterranen Klima zu optimieren. Anhand der täglichen Wasserbilanz konnte jedoch festgestellt werden, dass das System sehr stark von den klimatischen Verhältnissen abhängig ist.

Neben der Bereitstellung von Regenwasser für „Nicht-Trinkwasser“-Anwendungen kommen dezentrale Regenwasseranlagen auch vermehrt für den gezielten Rückhalt von Niederschlagswasser zum Einsatz (Huang et al. 2015; Sample and Liu 2014). Dezentrale Anlagenteile in der Siedlungsentwässerung, auch Low Impact Development (LID) genannt, werden verwendet, um Gesamtabflussmengen, Spitzenabflüsse sowie Überflutungsdauer bzw. -häufigkeit $\mathrm{zu}$ reduzieren und die Wasserqualität in angrenzenden Gewässern und Grundwasserkörpern $\mathrm{zu}$ verbessern. Diese Ziele werden durch die jeweilige Erhöhung von Evaporation, Infiltration, Speichervolumen sowie der Reinigungswirkung erreicht (Prince George's County 1991). Zu den dezentralen Anlagenteilen zählen beispielsweise auch Regentonnen, die ein temporäres Speichervolumen für den Rückhalt von Niederschlagswasser bereitstellen. Zudem ermöglichen Regentonnen die Nutzung von Regenwasser für „NichtTrinkwasser"-Anwendungen, wodurch sie für eine multifunktionale Regenwasserbewirtschaftung eingesetzt werden können. Jedoch ist eine erweiterte und vollständige Sichtweise des urbanen Wasserkreislaufes notwendig, um solche Anlagen auch effizient betreiben zu können (Sitzenfrei 2015). Zudem sind bei einer multifunktionalen Anwendung die Anforderungen an die zeitliche Auflösung von Messdaten und Simulationszeitschritten für die Bemessung und Überwachung höher. Campisano and Modica (2015) untersuchten die notwendige zeitliche Auflösung bei einer Regenwasserbewirtschaftung durch eine Variation der Simulationszeitschritte zwischen $5 \mathrm{~min}$ und $24 \mathrm{~h}$. Dabei wurde der Fokus auf die Effizienz in der Trinkwassereinsparung sowie die Retention von Niederschlagswasser gelegt. Es konnte festgestellt werden, dass für die Trinkwassereinsparung tägliche Zeitschritte ausreichend sind, während für eine Bewertung der Retentionswirkung mindestens Stundenwerte angewendet werden sollten. Besonders für kleine Retentionsvolumen sollten möglichst kleine Schrittweiten (idealerweise Minutenwerte) verwendet werden, um bei Regenereignissen mit hoher Intensität die Spitzenabflüsse besser reduzieren zu können (Oberascher et al. 2019). Jedoch sind derzeit für eine ausreichende Bewertung der multifunktionalen Anwendbarkeit noch immer empirische Daten erforderlich (Campisano et al. 2017). Des Weiteren wurde von Sample and Liu (2014) festgestellt, dass durch höhere Entnahmemengen für „Nicht-Trinkwasser“-Anwendungen die Rückhaltekapazität von ungesteuerten, dezentralen Anlagenteilen deutlich erhöht wird.

Durch die fortschreitende Digitalisierung im Bereich Siedlungswasserwirtschaft ergeben sich auch neue Möglichkeiten für die Überwachung und Steuerung von dezentralen Anlagenteilen in Echtzeit. Durch die vermehrte Einbindung von kostengünstigen Informations- und Kommunikationstechnologien (ICT) (z. B. Füllstandsmessgeräte mit Fernauslesung) ist der aktuelle $\mathrm{Zu}$ stand des Systems (fast) in Echtzeit bekannt. Beispielsweise verwendeten Kerkez et al. (2016) kostengünstige Sensoren und Steuerungsgeräte für den urbanen Wasserkreislauf und untersuchten, wie durch die Vielzahl an Messdaten die negativen Umwelteinflüsse reduziert werden können. In weiterer Folge können die sowohl räumlich als auch zeitlich hochaufgelösten Daten für die Umsetzung von mittel- und langfristigen Managementmaßnahmen in der Wasserversorgung beziehungsweise Siedlungsentwässerung verwendet werden. Wesentlich ist dabei der Einsatz numerischer Modelle, in denen die
Wirksamkeit verschiedenster Strategien modelltechnisch abgebildet werden kann (Sitzenfrei and Rauch 2014). Di Matteo et al. (2019) zeigten in einem simulationsbasierten Optimierungsprozess, dass durch das intelligente Öffnen und Schließen von Ventilen bei Speichervolumen die Spitzenabflüsse im Kanalsystem reduziert werden können. Oberascher et al. (2019) untersuchten das Potenzial von echtzeitgesteuerten Regentonnen und stellten fest, dass bereits einfache Kontrollstrategien eine Verbesserung des Gesamtsystems bewirken können.

Durch die Kombination von dezentralen Anlagen zur urbanen Wasserbewirtschaftung und von sich im Zuge der Digitalisierung rasant weiterentwickelnden ICT ergeben sich neue Anwendungen für den Einsatz kleiner Speichereinheiten, wie zum Beispiel „smarte“ Regentonnen. Die smarten Regentonnen (SRT) stellen primär in Echtzeit kontrollierbare Speichereinheiten für den Rückhalt von Niederschlagswasser dar und werden in dieser Arbeit näher vorgestellt. SRT werden auf Haushaltsebene installiert, wodurch das zurückgehaltene Niederschlagswasser sekundär auch für Bewässerungszwecke eingesetzt werden kann. Bei der SRT wird der Ausfluss automatisch gesteuert, wodurch die SRT vor Niederschlagsbeginn entleert werden kann, um zusätzliches Retentionsvolumen bereit zu stellen. Durch diese Möglichkeit wird die Effizienz der SRT für die Siedlungsentwässerung nicht von den vorherrschenden klimatischen Verhältnissen sowie den Entnahmen für Bewässerungszwecke beeinflusst. In dieser Arbeit werden zudem die Auswirkungen der SRT auf die Wasserversorgung sowie die Siedlungsentwässerung anhand eines zweistufigen Ansatzes untersucht. Im ersten Schritt wird ein reales Versuchsmodell der SRT vorgestellt. Das Versuchsmodell wurde bereits im Labormaßstab erprobt und wird im Frühjahr 2019 am Campus Technik der Universität Innsbruck zum Einsatz kommen. Im zweiten Schritt wird eine Wirksamkeitsanalyse auf Haushaltsebene mithilfe numerischer Simulationen durchgeführt. Dazu wurde eine 1-jährliche Regenserie in Kombination mit historischen Wettervorhersagen von der Zentralanstalt für Meteorologie und Geodynamik (ZAMG) verwendet. Die Niederschlagsdaten sind in einem Intervall von $1 \mathrm{~min}$ und die Wettervorhersagen in $15 \mathrm{~min}$ 


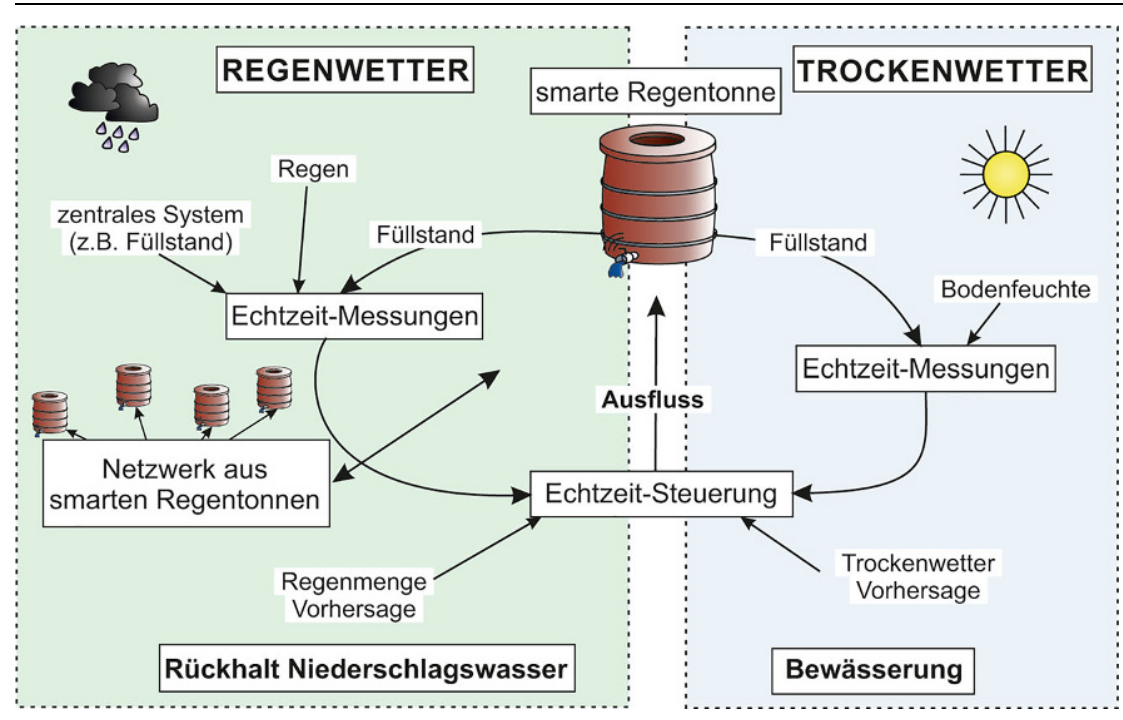

Abb. 1 Überblick über das Konzept der SRT und ihre Anwendungsmöglichkeiten in der Siedlungswasserwirtschaft mit Unterscheidung zwischen „Regenwetter" und „Trockenwetter"

Schritten verfügbar, wodurch auch die Effizienz kleiner Speichereinheiten, wie beispielsweise Regentonnen, simuliert werden kann. Eine solche Genauigkeit der Daten ist zudem für eine zielgerichtete Echtzeitsteuerung notwendig. Die Echtzeitsteuerungen für die SRT beinhalten unter anderem Kontrollstrategien für die Bewässerung (Reduktion von Trinkwasserverbrauch) sowie für den Rückhalt von Niederschlagswasser (Reduktion des Spitzenabflusses). Zusätzlich wird das Speichervolumen zwischen 0,1 und $1,0 \mathrm{~m}^{3}$ variiert, um das optimale Volumen unter Berücksichtigung der Wassereffizienz (Trinkwassereinsparung) und Regenwasserkontrolle (Überflutungsreduktion und Emissionskontrolle in Mischwasserüberlaufbauwerken) zu ermitteln. Die Ergebnisse zeigen, dass durch die Verwendung der SRT sowohl signifikante Trinkwassereinsparungen als auch Abflussreduktionen erzielt werden können. Eine einfache Abschätzung des vorhergesagten Zuflusses zur SRT, ohne Berücksichtigung des Vorhersagefehlers, ist jedoch nur bedingt geeignet, um am Ende des Niederschlagsereignisses die gewünschte Vollfüllung der SRT zu erzielen. Außerdem lässt die Betrachtung auf Haushaltsebene nur bedingt Aussagen über die Wirksamkeit im Kanalsystem zu. Für weitere Arbeiten ist es deshalb erforderlich, Kanalsystem und SRT gemeinsam zu betrachten, um die Auswirkung auf den Spitzenabfluss im Kanalsystem betrachten zu können.
Werden die SRT großflächig in einem Siedlungsgebiet implementiert, steht durch die große Anzahl ein relevantes, zusätzliches Retentionsvolumen zur Verfügung. Nach ÖWAV-Regelblatt 19 (2007) ist ein Nutzinhalt von $15 \mathrm{~m}^{3} / \mathrm{ha}_{\mathrm{Au}}$ für ein Mischwasserüberlaufbauwerk erforderlich. Ein durchschnittliches Einfamilienhaus hat eine Dachfläche von $165 \mathrm{~m}^{2}$ und bei einem Abflussbeiwert von 0,9 eine reduzierte Fläche von $150 \mathrm{~m}^{2}$. Werden beispielsweise 66 Einfamilienhäuser (reduzierte Gesamtfläche entspricht $1 \mathrm{ha}$ ) mit einer SRT und jeweils einem Volumen von 2501 ausgestattet, können $16,5 \mathrm{~m}^{3}$ an zusätzlichem Retentionsvolumen ohne hohen Bauaufwand bereitgestellt werden. Jedoch sind neue Anreize (zum Beispiel Fördermodelle, Bewilligungsverfahren für sowie auch Betreiber notwendig, damit diese Maßnahmen unterstützt und in zukünftigen Forschungstätigkeiten weiter thematisiert werden.

\section{Material und Methoden}

Das grundlegende Konzept der SRT, ihre Anwendungen in der Siedlungsentwässerung und der Trinkwasserversorgung sind in Abb. 1 dargestellt. Bei herkömmlichen Regentonnen wird üblicherweise erwartet, dass sie möglichst voll sind und somit der Bewässerungsbedarf gedeckt werden kann. Die SRT erfüllt dies ebenso und stellt zusätzlich eine in Echtzeit kontrollierbare Speichereinheit für eine multifunktionale Regenwasserzentrale Systeme) für die Bevölkerung bewirtschaftung dar. Zu diesem Zweck wird der Ausfluss aus der SRT durch ein automatisches Ventil gesteuert, welches bei Bedarf geöffnet beziehungsweise geschlossen wird. Die Entleerung kann beispielsweise vor dem Regenereignis in den Kanal aber auch in Versickerungsflächen erfolgen. Vor erwartetem Regenwetter (Wettervorhersage) wird die SRT entleert und somit kann, im Vergleich zur herkömmlichen Betriebsweise, mehr Dachablauf in der SRT zurückgehalten und gespeichert werden, wodurch der Spitzenabfluss im Kanalnetz reduziert wird. Das gespeicherte Regenwasser kann wie üblich in darauffolgenden Trockenperioden für die Gartenbewässerung verwendet werden, um den Bedarf an Trinkwasser zu vermindern.

Die SRT stellen jedoch im Verhältnis zum Gesamtsystem der Siedlungsentwässerung ein kleines Speichervolumen dar. Für einen einfachen Größenvergleich betrachten wir eine Regentonne mit einem Volumen von 2001 und einer angeschlossenen Dachfläche von $100 \mathrm{~m}^{2}$. Dabei entspricht das Volumen der Regentonne einer Niederschlagsmenge von $2 \mathrm{~mm}$. Flächendeckend eine Niederschlagsmenge von bis $\mathrm{zu} \mathrm{zu}-$ sätzlich $2 \mathrm{~mm}$ zur richtigen Zeit zu speichern, kann einen signifikanten Mehrwert für die zentrale Siedlungsentwässerung bedeuten. Beispielsweise beträgt der Bemessungsniederschlag am Campus Technik der Universität Innsbruck (ÖKOSTRA Gitterpunkt 4737) für ein 2-jährliches Ereignis mit einer Dauer von $15 \mathrm{~min} 16,4 \mathrm{~mm}$, somit könnten bis zu $12 \%$ des Volumens beim Spitzenabflusses im zentralen Entwässerungssystem durch SRT verzögert werden. Für die Mischwasserbewirtschaftung kann ein Rückhalt von $2 \mathrm{~mm}$ Reduktionen von Mischwasserentlastungen bewirken. Um somit solch kleine Speichereinheiten für die Siedlungsentwässerung effizient nutzen $\mathrm{zu}$ können, sind neue Bewirtschaftungsstrategien, wie die Einbindung in eine smarte Wasserinfrastruktur und die Wettervorhersage, notwendig.

\subsection{Bewirtschaftungskonzept}

In Abhängigkeit von der Wettervorhersage wird der prognostizierte Niederschlag ermittelt und der Zufluss zur SRT abgeschätzt. Es wird von einer teilgefüllten SRT ausgegangen, welche bereits durch vergangene Niederschlagsereignisse gefüllt wurde. Daher 
wird zwischen den Begriffen Retentionsvolumen, Speichervolumen und Gesamtvolumen unterschieden und wie folgt definiert:

$$
\begin{aligned}
& \text { Gesamtvolumen }^{\mathrm{SRT}} \\
= & \text { Retentionsvolumen }^{\mathrm{SRT}} \\
& + \text { Speichervolumen }^{\mathrm{SRT}}
\end{aligned}
$$

Mit dem Begriff Speichervolumen wird das Volumen des gespeicherten Regenwassers bezeichnet. Der Begriff Retentionsvolumen definiert das noch vorhandene Restvolumen zwischen der Teilfüllungsmenge und dem Gesamtvolumen der SRT. Beispielsweise ist bei einer vollständig gefüllten Regentonne kein Retentionsvolumen vorhanden, während das Speichervolumen dem Gesamtvolumen entspricht. Im umgekehrten Fall, bei einer komplett entleerten Regentonne, entspricht das Retentionsvolumen dem Gesamtvolumen und das Speichervolumen ist null.

Der abgeschätzte Zufluss zur SRT wird mit dem vorhandenen Retentionsvolumen der SRT verglichen, wodurch sich für den Fall „Regenwetter“ drei unterschiedliche Kontrollstrategien ergeben. In der ersten Kontrollstrategie ist der prognostizierte Zufluss zur SRT kleiner als das vorhandene Retentionsvolumen. Da die SRT auch nach dem Niederschlagsereignis noch nicht vollgefüllt ist, ist in dieser Kontrollstrategie keine weitere Maßnahme erforderlich. Ist hingegen der prognostizierte Zufluss zur SRT größer als das vorhandene Retentionsvolumen, aber kleiner als das Gesamtvolumen, kommt die zweite Kontrollstrategie zur Anwendung. Dabei wird vor Niederschlagsbeginn das Ventil der SRT automatisch geöffnet und die SRT beginnt sich zu entleeren. Wenn das Retentionsvolumen der vorhergesagten Zuflussmenge entspricht, wird das Ventil wieder geschlossen. Dadurch wird erreicht, dass der gesamte, erwartete Niederschlag von der SRT zurückgehalten wird. Zusätzlich ist die SRT nach Niederschlagsende (möglichst) vollständig gefüllt, um in Bezug auf die Bewässerung und Trinkwassereinsparung bestmögliche Effizienz $\mathrm{zu}$ erreichen. In der dritten Kontrollstrategie ist der prognostizierte Zufluss höher als das Gesamtvolumen der SRT. Daher wird die SRT vor Beginn des Niederschlagsereignisses vollständig entleert, um das Gesamtvolumen der SRT als Retentionsvolumen nutzen zu können. Durch die Einbindung der Wettervorhersage ist die zukünftige Wetterent- wicklung durch die Niederschlagsmenge und der zeitliche Verlauf der Niederschlagsintensität während des Regenereignisses zumindest teilweise bekannt. Zudem stehen durch die Verwendung in einer smarten Wasserinfrastruktur eine Vielzahl an hochaufgelösten Messwerten, im Idealfall Minutenwerte, zur Verfügung. Durch Messungen von Füllständen in Schächten, Versickerungsmulden und Rückhaltebecken sind die aktuellen Systemzustände im Kanalnetz bekannt. Diese Informationen können dann dazu verwendet werden, um das Retentionsvolumen der SRT bei Niederschlagsereignissen, bei denen der Zufluss das Gesamtvolumen der Regentonne überschreitet, bestmöglich einzusetzen. Zum Beispiel wird die SRT erst kurz vor dem Auftreten des Spitzenabflusses eingesetzt, um den Spitzenabfluss im Kanal und dadurch auch Überlastungen zu reduzieren. Idealerweise steht ein Netzwerk von über das Einzugsgebiet verteilten SRT zur Verfügung, die gemeinsam gesteuert werden. Auch kann durch aktuelle Niederschlagsmessungen die vorhergesagte Wetterentwicklung überprüft und es können, falls erforderlich, die Kontrollparameter angepasst werden (zum Beispiel durch Machine Learning).

Für den Fall „Trockenwetter“ kommt eine zusätzliche Kontrollstrategie zur Anwendung. Das zurückgehaltene Niederschlagswasser wird nun für die Bewässerung von Grünflächen, zum Beispiel Gärten oder grüner Infrastruktur, verwendet. Dazu werden Messungen der Bodenfeuchte in Grünflächen verwendet. In Abhängigkeit von der Bodenfeuchte kann festgestellt werden, ob eine Bewässerung der Grünflächen erforderlich ist. Im Falle einer Bewässerung beginnt die SRT zu bewässern und nachdem der Bedarf (z.B. durch Erreichen einer bestimmten Bodenfeuchte) gedeckt ist, wird das Ventil der smarten Regentonne wieder automatisch geschlossen, um auch für weitere Trockentage die Bewässerungsmenge durch Regenwasser abdecken zu können. Für den Fall, dass der Bewässerungsbedarf nicht durch die SRT gedeckt werden kann, kann Trinkwasser für die Bewässerung aus dem Wasserversorgungssystem entnommen werden.

Für die Umsetzung des SRT-Konzeptes wurde ein zweistufiger Ansatz verfolgt. Um die Auswirkungen der SRT auf die Wasserversorgung sowie auf die Siedlungsentwässerung zu demonstrieren, wurden numerische Simula- tionen für den Wirksamkeitsnachweis auf Haushaltsebene durchgeführt. Des Weiteren wurde ein Versuchsmodell der SRT gebaut und die Funktionsfähigkeit im Labormaßstab geprüft.

\subsection{Versuchsmodell}

Für Demonstrationszwecke wurde mit der Entwicklung eines Versuchsmodells der SRT begonnen. Regentonnen benötigen nur wenig Platz, wodurch sie auch nachträglich in die bestehende Entwässerungsinfrastruktur integriert werden können. Die Verwendung einer herkömmlichen Regentonne, wie sie in Baumärkten erhältlich ist, wurde als wesentlicher Punkt angesehen. Durch die Ausstattung mit einer Steuerungsmöglichkeit wurde die herkömmliche Regentonne in eine SRT umgewandelt. Während der Entwicklung wurde auch ein Modell im Labormaßstab umgesetzt und die Grundfunktionsfähigkeit getestet. Die Ergebnisse aus den Labortests wurden anschließend für die Entwicklung des Versuchsmodells verwendet.

Ein weiterer Anforderungspunkt an die Umsetzung war die Einbindung in eine smarte Wasserinfrastruktur. Der Campus Technik der Universität Innsbruck wurde im Zuge des Sondierungsprojekts „Smart Water Control" mit einem innovativen Mess- und Steuerungsnetzwerk für die Wasserversorgung, Abwasserentsorgung sowie für die Siedlungsentwässerung ausgestattet. Am "Smart Campus“ werden beispielsweise Wasserverbräuche, Niederschlag und Füllstände im Kanal in Minutenintervallen gemessen und in eine zentrale Datenbank übertragen. Basierend auf den Messdaten werden alle Wasserströme zum und vom Campusgelände in Echtzeit überwacht. Daher stellt der Smart Campus ein ideales Experimentier- und Demonstrationsobjekt für innovative Anwendungen im Bereich der Siedlungswasserwirtschaft, wie zum Beispiel das Testen des SRT Konzeptes, dar.

In Abb. 2 ist das entwickelte und konstruierte Versuchsmodell der SRT erkennbar. Hauptelement ist dabei eine herkömmliche Regentonne, die als Speichereinheit für den Niederschlag dient. Das Speichervolumen dieser SRT entspricht 200l. Am Boden der SRT befinden sich ein automatisch und ein händisch steuerbares Ventil. Das automatisch gesteuerte Ventil wird für die kontrollierte Wasserentnahme, für die Bereitstellung von Retentionsvolumen 


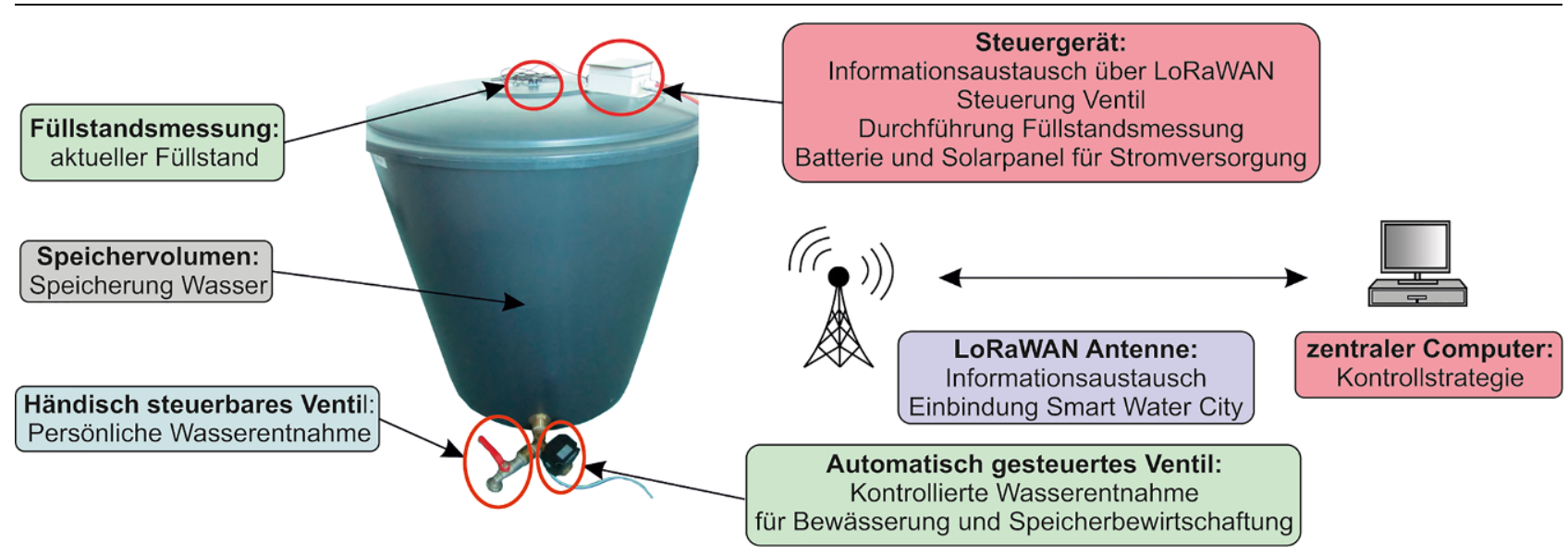

Abb. 2 Versuchsmodell der SRT und die Beschreibung der wesentlichen Bauteile

wie auch für die bedarfsabhängige Bewässerung von Grünflächen verwendet. Für die persönliche Wasserentnahme kann das händisch steuerbare Ventil verwendet werden. Im Deckel der smarten Regentonne ist zudem ein Füllstandsmessgerät eingebaut. Durch Kenntnis des aktuellen Füllstands kann der Füllungsgrad der SRT bestimmt werden. Die SRT wird über ein $\mathrm{Ge}$ rät gesteuert, das sich ebenfalls am Deckel befindet. Das Steuerungsgerät führt das Öffnen und Schließen des automatisch gesteuerten Ventils sowie die Füllstandsmessung durch und besitzt zudem eine eingebaute LoRaWAN-Antenne für den Informationsaustausch mit der Umgebung. Die Datenübertragungstechnik LoRaWAN gehört zu den Niedrigfunkenergienetzen und die Messdaten können über eine hohe Reichweite bei gleichzeitig geringem Energieverbrauch übermittelt werden (Mekki et al. 2018). Dadurch ist LoRaWAN besonders für batteriebetriebene Sensoren geeignet und wurde bereits von Blumensaat et al. (2017) für Messungen im Kanalsystem erfolgreich verwendet. Bei der SRT werden die Steuerungsbefehle für das Öffnen und Schließen des Ventils sowie die Füllstandsmessungen über LoRaWAN übertragen. Die Daten werden von einem zentralen Computer verarbeitet und durch die Einbindung der Wettervorhersage und unter Berücksichtigung der Messdaten vom Smart Campus kann die beste Kontrollstrategie ermittelt werden. Das im Labor erprobte Versuchsmodell wird im Frühjahr 2019 am Campusgelände der Universität Innsbruck aufgestellt und unter realen Bedingungen getestet.

\subsection{Numerische Simulation}

\subsubsection{Fallbeispiel Einfamilienhaus}

Für eine erste Wirkungsanalyse wurden die Auswirkungen der SRT auf Wasserversorgung und Siedlungsentwässerung jeweils auf Haushaltsebene untersucht und mit der Wirkungsweise von herkömmlichen Regentonnen verglichen. In Österreich werden ca. $90 \%$ der Gebäude für Wohnzwecke genutzt, wobei ca. $75 \%$ der Gebäude Einfamilienhäuser oder Wohngebäude mit 2 Wohnungen sind (Asamer et al. 2011). Daher wurden hypothetische Gebäude mit 1 oder 2 Wohnungen für die Implementierung der SRT ausgewählt. Im Jahr 2017 wurden 14.727 neue Wohngebäude mit 1 oder 2 Wohnungen mit einer überbauten Gesamtfläche von $2.430 .478 \mathrm{~m}^{2}$ in Österreich fertiggestellt (Statistik Austria 2018a). Dadurch ergibt sich eine überbaute Fläche von $165 \mathrm{~m}^{2}$ pro Wohngebäude mit 1 oder 2 Wohnungen. Weiters betrug 2017 die durchschnittliche Haushaltsgröße 2,22 Personen (Statistik Austria 2018b). Anhand dieser Daten wurde für die Wirksamkeitsanalyse auf Haushaltsebene ein Einfamilienhaus mit einer überbauten Fläche von $165 \mathrm{~m}^{2}$ und einer Haushaltsgröße von 2,2 Personen angenommen. Für das Fallbeispiel wurde das Volumen der SRT zwischen 0,1 und $1,0 \mathrm{~m}^{3}$ variiert.

\subsubsection{Wasserbedarf und Niederschlag}

Für die Ermittlung des Bewässerungsbedarfs wurde die statistische Auswertung über den österreichischen Wasserverbrauch von Neunteufel et al. (2012) verwendet. Da die Nutzung von Trink- wasser im Außenbereich ausschließlich im Sommerhalbjahr stattfindet, wurde für das Fallbeispiel die Bewässerungsperiode vom 21. März bis zum 22. September festgelegt. Des Weiteren kommt es vorwiegend im Sommerhalbjahr zu Mischwasserüberlaufen in Österreich, die durch Starkregenereignisse verursacht werden (Kleidorfer et al. 2009). Wie Neunteufel et al. (2012) außerdem feststellten, hat die mittlere Tagestemperatur den wesentlichsten Einfluss auf die im Außenbereich verwendete Wassermenge, welche mit zunehmender Tagestemperatur deutlich ansteigt. Für die Ermittlung der Außenbedarfsmenge wurden die von Neunteufel et al. (2012) erhobenen Werte (Tab. 1) in Abhängigkeit von der mittleren Tagestemperatur verwendet.

Um die mittleren Tagestemperaturen bestimmen zu können, wurden Wetterdaten der Wetterstation Innsbruck Flughafen aus dem Jahr 2015 verwendet. Die Wetterstation befindet sich im Süden des Flughafens von Innsbruck und wird von der Zentralanstalt für Meteorologie und Geodynamik (ZAMG) betrieben. Die Temperaturmesswerte sind in 10-min-Intervallen erhältlich, aus denen die mittlere Tagestemperatur ermittelt wurde. Anschließend wurden die mittleren Tagestemperaturen den in Tab. 1 angeführten, durchschnittlichen Außenverbräuchen zugeordnet. Der Verlauf der mittleren Tagestemperatur kann der roten Kurve in Abb. 3 entnommen werden. $\mathrm{Zu}$ Beginn der Bewässerungsperiode liegt die mittlere Tagestemperatur unter $10^{\circ} \mathrm{C}$, wodurch auch kein Außenverbrauch stattfindet. Im Zeitraum zwischen Mitte April und Anfang Juli schwankt die Tagestemperatur zwischen 10 und $25^{\circ} \mathrm{C}$ und der 
Tab. 1 Bewässerungsbedarf pro Tag und Person $(I /(d \cdot P))$ in Abhängigkeit von der mittleren Tagestemperatur. (Nach Neunteufel et al. 2012)

\begin{tabular}{|l|l|l|}
\hline $\begin{array}{l}\text { Mittlere Tages- } \\
\text { temperatur }\left({ }^{\circ} \mathrm{C}\right)\end{array}$ & $\begin{array}{l}\text { Anzahl } \\
\text { Tage 2015 } \\
{[-]}\end{array}$ & $\begin{array}{l}\text { Durchschnitt- } \\
\text { licher Außen- } \\
\text { verbrauch } \\
(\mathrm{l} /(\mathrm{d} \cdot \mathrm{P}))\end{array}$ \\
\hline $\mathrm{T}<10$ & 28 & 0 \\
\hline $10 \leq \mathrm{T}<15$ & 51 & 8 \\
\hline $15 \leq \mathrm{T}<20$ & 59 & 32 \\
\hline $20 \leq \mathrm{T}<25$ & 46 & 75 \\
\hline $25 \leq \mathrm{T}$ & 3 & 140
\end{tabular}

Außenverbrauch beträgt zwischen 8 und $75 \mathrm{l} /(\mathrm{d} \cdot \mathrm{P})$. Im Juli und August liegt die mittlere Tagestemperatur meistens zwischen 15 und $25^{\circ} \mathrm{C}$, zudem wird im Juli an 3 Tagen die mittlere Tagestemperatur von $25^{\circ} \mathrm{C}$ überschritten, bei denen ein Außenverbrauch von 1401/(d-P) benötigt wird. Im September nimmt die mittlere Tagestemperatur wieder ab und beträgt meistens zwischen 10 und $20^{\circ} \mathrm{C}$.

Für den Niederschlag wurden die entsprechenden Messdaten derselben Wetterstation (2015, Innsbruck Flughafen, Quelle: ZAMG) verwendet. Die Regendaten lagen in 1-min-Intervallen vor und der aufsummierte Gesamtniederschlag für das Jahr 2015 betrug $852,4 \mathrm{~mm}$.

Für eine multifunktionale Regenwasserbewirtschaftung von ungesteuerten Speichereinheiten (herkömmliche Regentonnen) ist eine kontinuierliche Wasserentnahme erforderlich, um Retentionsvolumen für Niederschlagsereignisse $\mathrm{zu}$ schaffen. Wasser im Außenbereich wird überwiegend im Sommerhalbjahr benötigt, das heißt, in diesem Zeitraum wird Regenwasser aus dem Speicher entnommen. Außerhalb des Sommerhalbjahrs ist kein Bedarf für eine Entnahme von Regenwasser gegeben, wodurch auch kein Niederschlagswasser zurückgehalten wird. Falls jedoch Regenwasser aus der herkömmlichen Regentonne entnommen würde, könnte das Volumen zur Retention genutzt werden. Daher wird für die Wirksamkeitsanalyse der Niederschlag in der Periode vom 21. März bis zum 23. September berücksichtigt. Für eine bessere Übersichtlichkeit wurden die Niederschläge auf Tageswerte aufsummiert und in Abb. 3 dargestellt. Die Tagesniederschläge schwanken dabei zwischen $0 \mathrm{~mm} / \mathrm{d}$ und max. $45 \mathrm{~mm} / \mathrm{d}$. Der Gesamtniederschlag während des Sommerhalbjahrs 2015 betrug $651,7 \mathrm{~mm}$.
Im Gegensatz zu herkömmlichen Regentonnen können SRT durch die automatische Entleerung für den Rückhalt von Niederschlagswasser auch außerhalb des Sommerhalbjahrs eingesetzt werden, da sie nicht auf die Entnahme von Trinkwasser durch die Deckung des Außenbedarfs angewiesen sind. Um jedoch die Ergebnisse vergleichbar zu machen, wurde bei der SRT ebenfalls nur das Sommerhalbjahr berücksichtigt.

Für die Berücksichtigung der Wetterentwicklung wurden reale (historische) Wettervorhersagen der ZAMG für dieselbe Periode aus dem Integrated Nowcasting through Comprehensive Analysis (INCA)-System verwendet. Aufbauend auf numerischen Wettermodellen werden Daten aus Wetterstationen und Radarmessungen in das INCA-System integriert, um die Qualität der Wettervorhersagen für „Nowcasting“ (0-4h) und kurze Vorhersagezeiträume (bis $12 \mathrm{~h}) \mathrm{zu}$ verbessern. Im INCA-System wird Österreich in ein Netz mit einer horizontalen Auflösung von $1 \times 1 \mathrm{~km}$ unterteilt und die unterschiedliche Topografie wird durch einen Höhenwert berücksichtigt (Haiden et al. 2011). Die historischen Wettervorhersagen für das Jahr 2015 lagen in 15-min-Schritten über einen Zeitraum von jeweils $24 \mathrm{~h}$ vor. In der numerischen Simulation wurden verschiedene Vorhersagezeiträume, in weiterer Folge „Akkumulationszeit“ genannt, in einem Intervall von $1-24 \mathrm{~h}$ verwendet.

\subsubsection{Kontrollstrategie}

Für eine erste Wirksamkeitsanalyse der SRT auf Haushaltsebene wurde eine einfache Steuerung umgesetzt. Die einzelnen Kontrollschritte sind in Abb. 4 beschrieben. Für die beiden Ziele, die Bereitstellung von Regenwasser für die Substitution von Trinkwasser und die Retention von Niederschlagswasser, kommen unterschiedliche Kontrollschritte zur Anwendung, welche sich in weiterer Folge in verschiedene Analyse- und Ausführungszeiträume einteilen lassen. In der Umsetzung wird der Ausfluss aus der SRT über ein Ventil gesteuert.

Die EN 16941-1 (2018) empfiehlt, für Wirksamkeitsberechnungen von Anlagen für Regenwassernutzung den Ertrag am Ende des gewählten Zeitschrittes zu ermitteln. Die Bewässerung erfolgt einmal täglich, deshalb wird im dargestellten Fallbeispiel das Regenwasser für die Bewässerung am Ende jedes einzelnen Tages aus der SRT entnommen. Um 23:00 Uhr wird der Bewässerungsbedarf aus der mittleren Tagestemperatur und der Anzahl der Personen im Haushalt berechnet. Anschließend wird der Füllstand in der SRT und in weiterer Folge das Volumen des gespeicherten Niederschlagswassers ermittelt. Ist die SRT leer, kann kein Niederschlagswasser aus der SRT entnommen werden und der gesamte Bewässerungsbedarf wird aus dem Trinkwassersystem entnommen. Ist hingegen die SRT gefüllt, wird beurteilt, ob der Bewässerungsbedarf durch das gespeicherte Regenwasser gedeckt werden kann. Kann der Bewässerungsbedarf nicht gedeckt werden, wird zuerst Regenwasser aus der SRT entnommen und anschließend der restliche Bewässerungsbedarf durch Trinkwasser gedeckt. Bei einer vollständigen Deckung übernimmt die SRT die Versorgung für den Bewässerungsbedarf. In beiden Fällen wird das Zielvolumen (leere Tonne oder gespeichertes Niederschlagsvolumen abzüglich Bewässerungsbedarf) festgelegt und das Ventil wird geöffnet, wodurch sich die SRT zu entleeren beginnt. Anschließend wird in jedem Simulationsschritt beurteilt, ob das Zielvolumen erreicht ist, und nach Erreichen des Zielvolumens wird das Ventil wieder geschlossen.

Ein wesentliches Element für die Retention von Niederschlagswasser stellt die Einbindung der Wetterentwicklung dar. Im ersten Schritt wird die prognostizierte Niederschlagsmenge für die gewählte Akkumulationszeit bestimmt. Der Analyse- bzw. Ausführungszeitschritt entspricht dabei der halben Akkumulationszeit. Beträgt die Akkumulationszeit z.B. $1 \mathrm{~h}$, wird alle $30 \mathrm{~min}$ die prognostizierte Niederschlagsmenge für die nächsten $60 \mathrm{~min}$ ermittelt. In weiterer Folge wird der tatsächlich gefallene Niederschlag während jedes Analysezeitschritts in der Simulation berücksichtigt. Dabei wird von der prognostizierten Niederschlagsmenge der bereits gefallene Niederschlag abgezogen, da die SRT in Abhängigkeit vom Zufluss gesteuert wird. Im Anschluss wird die noch $\mathrm{zu}$ erwartende Niederschlagsmenge mit der Einzugsfläche und einem Abflussbeiwert von 0,9 multipliziert (DWA-M 153 2007), um den weiteren Zufluss zur smarten Regentonne abzuschätzen. Dieser geschätzte Zufluss wird in weiterer Folge mit dem vorhandenen Retentionsvolumen verglichen. Wenn das vorhandene Retenti- 


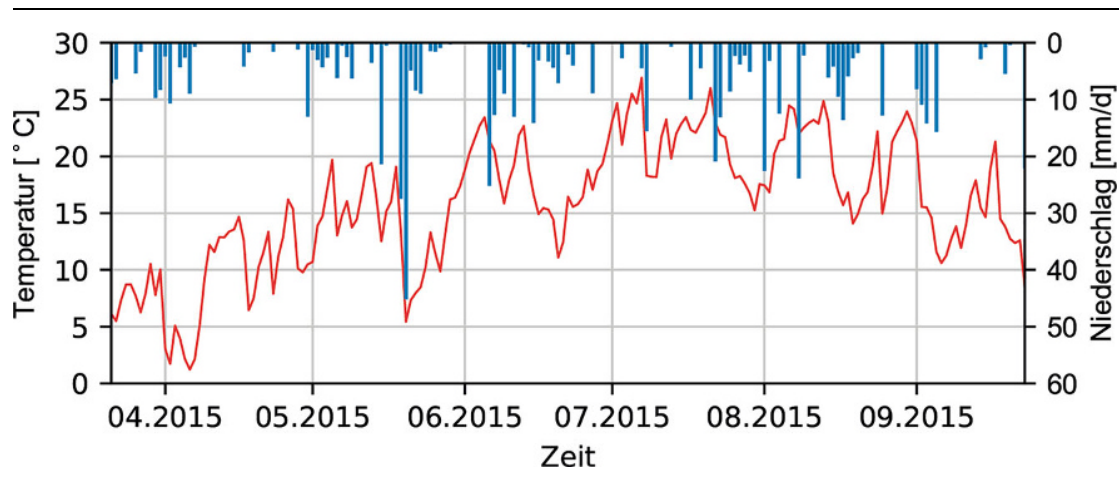

Abb. 3 Mittlere Tagestemperatur und aufsummierter Tagesniederschlag während der Bewässerungsperiode

onsvolumen größer als der Zufluss ist, ist keine Aktion erforderlich. Ist das vorhandene Retentionsvolumen hingegen kleiner als der geschätzte Zufluss, wird das Ventil geöffnet und die SRT beginnt sich zu entleeren (in den Kanal oder in eine Versickerungsanlage). Anschließend wird in jedem Simulationsschritt überprüft, ob das Retentionsvolumen dem abgeschätzten Zufluss entspricht und bei Bedarf wird das Ventil geschlossen.

\subsubsection{Numerische Simulation}

Der Dachabfluss vom Einfamilienhaus wurde durch eine hydrodynamische Simulation ermittelt. Dazu wurde eine Eingabedatei für die Software EPA Storm Water Management Model, Versionsnummer 5.1.012, (SWMM5) (Rossman 2015) erstellt. Die Eingabedatei beinhaltete eine Einzugsfläche für die Dachfläche und einen Netzknoten, der den Anschluss an das öffentliche Kanalnetz darstellt. Zwischen der Einzugsfläche und dem Netzknoten wurde die SRT als LID-Objekt mit einem Speichervolumen eingefügt. Für die Regulierung der Ausflussmenge aus der SRT wurde der Drainagekoeffizient verwendet, der in SWMM5 einen Beiwert für die Berechnung des Ausflusses aus LID-Objekten entspricht. Einen wesentlichen Einfluss auf die Simulationsergebnisse hat die Entleerungszeit der SRT. Um die Ergebnisse daher vergleichbar zu machen, wurde unabhängig vom Volumen der Regentonne eine einheitliche Entleerungszeit verwendet. Dabei wurde vorausgesetzt, dass die SRT auch beim kürzesten Analysezeitschritt - welcher im Fallbeispiel 30 min beträgt - vollständig geleert werden kann. Dadurch wurde auch die Entleerungszeit mit $30 \mathrm{~min}$ angesetzt und entspricht einem
Drainagekoeffizienten von $85 \mathrm{~mm} / \mathrm{h}$ in SWMM5.

Für die Ausführung der hydrodynamischen Simulation wurde PySWMM (https://github.com/jennwuu/ pyswmm) verwendet. PySWMM ist ein Python Wrapper für SWMM5 und verwendet den Berechnungsalgorithmus von SWMM5. Jedoch erlaubt PySWMM eine Schritt-für-Schritt-Simulation von SWMM5-Eingabedateien, wodurch eine Vielzahl an Kontrollmöglichkeiten umgesetzt werden kann. Beispielsweise kann dadurch die Wettervorhersage in die Kontrollstrategie eingebunden werden. Ein weiterer Vorteil von PySWMM ist, dass auch kleine Speichervolumen $\left(<1,0 \mathrm{~m}^{3}\right)$ in Echtzeit gesteuert werden können. Im Falle der SRT wird der Drainagekoeffizient verändert und je nach Bedarf entweder auf 0 (kein Ausfluss) oder auf den Drainagekoeffizienten von $85 \mathrm{~mm} / \mathrm{h}$ gesetzt, der eine vollständiSimulationszeitraum wurde entsprechend der Bewässerungsperiode (Sommerhalbjahr: 21. März-23. September) angesetzt. Für die Wirksamkeitsanalyse wurden 10 verschiedene Regentonnengrößen $\left(0,1-1,0 \mathrm{~m}^{3}\right)$ und 3 Akkumulationszeiten $(1 \mathrm{~h}, 4 \mathrm{~h}$ und $12 \mathrm{~h})$ mit dem Referenzwert, der herkömmlichen Regentonne, verglichen. Die insgesamt 40 Simulationen wurden automatisiert durchgeführt und ausgewertet.

\section{Ergebnisse und Diskussion}

\subsection{Beispiel Funktionsweise SRT}

Abb. 5 veranschaulicht die grundlegende Funktionsweise der SRT anhand eines Beispiels für ein Regenereignis am 25. Juli 2015. Dazu wurde eine SRT mit einem Volumen von $0,5 \mathrm{~m}^{3}$, einer Akkumulationszeit von $60 \mathrm{~min}$ sowie einem Analysezeitschritt von $30 \mathrm{~min}$ ge Entleerung in $30 \mathrm{~min}$ erlaubt. Der verwendet. Zudem wurde eine perfekte Wettervorhersage angenommen (Vorhersage entspricht exakt dem tatsächlichen Regenereignis), um die ideale Funktionsweise darstellen zu können. Als Eingangsparameter für die Steuerung dienen die Niederschlagsmengen ( $\mathrm{mm} / \mathrm{min}$, blaue Balken) und der Abfluss von der Dachfläche (l/s) zur SRT (blau strichlierte Linie). Zudem ist das aktuelle Speichervolumen $\left(\mathrm{m}^{3}\right)$ innerhalb der SRT durch die rot gestrichelte Linie erkennbar und die Ausflussmengen $(1 / \mathrm{s})$ aus der SRT in das Kanalsystem können der roten durchgehenden Linie entnommen werden.

Zum ersten Analysezeitpunkt um 12:00 Uhr ist die SRT durch ein vorheriges Niederschlagsereignis bereits teilgefüllt und das Speichervolumen beträgt $0,22 \mathrm{~m}^{3}$. Für die nächsten $60 \mathrm{~min}$ wurde kein Niederschlag prognostiziert, wodurch keine weitere Aktion bis zum nächsten Analysezeitpunkt erforderlich war. Um 12:30 Uhr wurde die nächste Analyse durchgeführt und die prognostizierte Niederschlagsmenge für die nächsten $60 \mathrm{~min}$ (13:30 Uhr) betrug $3,0 \mathrm{~mm}$. Durch eine Fläche von $165 \mathrm{~m}^{2}$ und einen Abflussbeiwert von 0,9 wurde der Zufluss zur SRT mit $0,45 \mathrm{~m}^{3}$ abgeschätzt. Da zu diesem Zeitpunkt nur ein Retentionsvolumen von $0,28 \mathrm{~m}^{3}$ zur Verfügung stand, wurde das Ventil geöffnet und die SRT begann sich zu entleeren. Um 12:44 Uhr wurde in der SRT ein Volumen von $0,05 \mathrm{~m}^{3}$ gemessen und das Ventil wurde geschlossen, da das erforderliche Retentionsvolumen von $0,45 \mathrm{~m}^{3}$ erreicht wurde. Bis zum nächsten Analysezeitpunkt um 13:00 Uhr wurde kein Niederschlag gemessen, wodurch sich auch das Speichervolumen nicht veränderte. Die Auswertung um 13:00 Uhr ergab eine neu prognostizierte Niederschlagsmenge von $3,7 \mathrm{~mm}$ und einen abgeschätzten $\mathrm{Zu}$ fluss von $0,55 \mathrm{~m}^{3}$. Da der abgeschätzte Zufluss höher als das Gesamtvolumen der SRT ist, wurde die SRT komplett entleert. Um 13:15 Uhr setzte Niederschlag mit einer maximalen Intensität von $0,3 \mathrm{~mm} / \mathrm{min}$ ein und die SRT begann sich zu füllen. Die Vollfüllung wurde um 13:33 Uhr erreicht. Die weitere Abfolge von Entleerungs- und Füllungsphasen der SRT ist anhand des Speichervolumens gut erkennbar. Während der Periode von 12:00-19:00 Uhr sind drei deutliche Entleerungsphasen vor Regenbeginn erkennbar, durch die zusätzliches Retentionsvolumen bereitgestellt wird. Zudem konnte durch die 


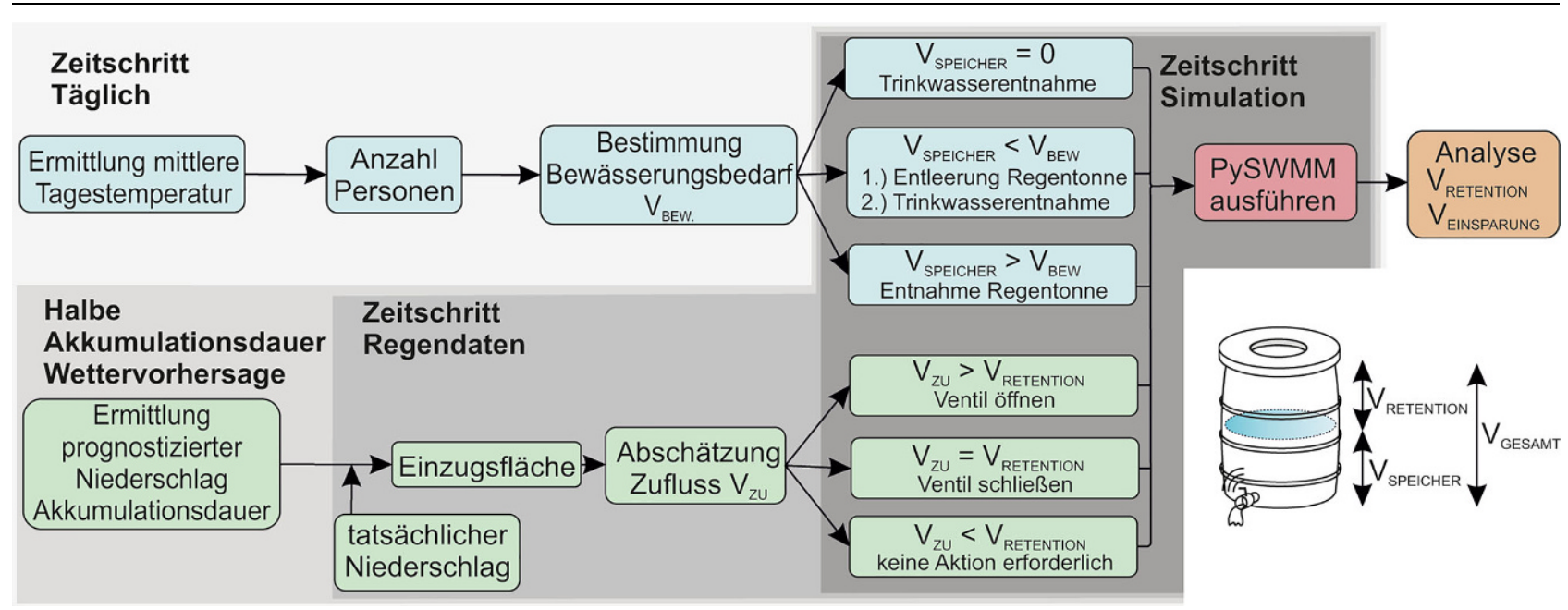

Abb. 4 Kontrollschritte und Analyse- und Ausführungszeiträume für die Bewässerung sowie für den Rückhalt von Niederschlagswasser

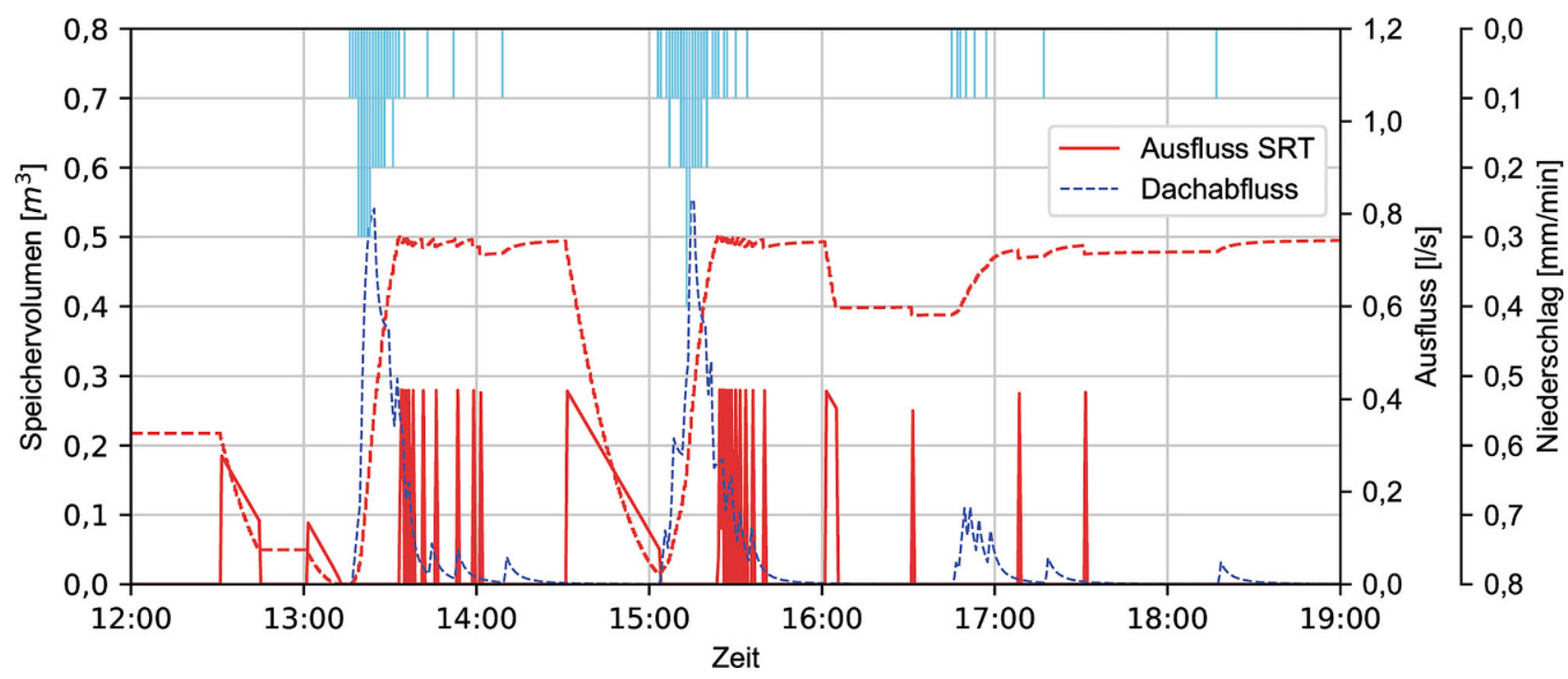

Abb. 5 Niederschlag, Dachabfluss, Speichervolumen sowie Ausfluss aus der smarten Regentonne für ein Regenereignis am 25. Juli 2015: Niederschlag [mm/min] (blaue Balken), Abfluss Dachflächezur SRT [l/s] (blau strichlierte Linie), aktuelles Speichervolumen SRT $\left[\mathrm{m}^{3}\right]$ (rot gestrichelte Linie), Ausflussmengen SRT [l/s] (rote Linie)

SRT der Spitzenabfluss in das Kanalsystem von $0,831 /$ s (Dachabfluss) auf $0,411 /$ s reduziert und verzögert werden.

\subsection{Systematische Analyse}

Für die systematische Analyse wurden die realen, historischen Wettervorhersagen verwendet, wodurch auch Unsicherheiten in den Wettervorhersagen einen Einfluss auf die Wirksamkeit der SRT haben. In Abb. 6 sind die Ergebnisse für die SRT auf Haushaltsebene für eine Jahressimulation ersichtlich. In der Simulation wurden das Volumen der SRT und die Akkumulationszeiten der Wettervorhersage variiert. Als Re- ferenzwert wurde eine herkömmliche Regentonne (siehe schwarze, durchgehende Line) verwendet, wobei aus der ungesteuerten Regentonne Wasser nur für Bewässerungszwecke entnommen wird. Abb. 6a zeigt die Einsparungen an Trinkwasser, während in Abb. 6b das Gesamtvolumen an zurückgehaltem Niederschlagswasser dargestellt ist. Die Abbildungen zeigen die unterschiedlichen Ergebnisse für die Akkumulationszeiten $1 \mathrm{~h}, 4 \mathrm{~h}$ und $12 \mathrm{~h}$. Zudem werden die jeweiligen Ergebnisse mit der maximal erreichbaren Genauigkeit, d.h. einer perfekten Wettervorhersage, verglichen.

\subsubsection{Einsparung Trinkwasser}

Wie erwartet, nimmt die Einsparung an Trinkwasser mit höheren Gesamtvolumen der SRT zu. Während der Bewässerungsperiode von 21. März bis 23. September ist eine Bewässerung an 159 Tagen mit einer Bewässerungsmenge von $13,6 \mathrm{~m}^{3}$ erforderlich. Anstatt Trinkwasser kann durch die SRT je nach Tonnengröße (zwischen 0,1 und $1,0 \mathrm{~m}^{3}$ ) und Akkumulationszeit (zwischen 1 und $12 \mathrm{~h}$ ) zwischen 2,1 und $11,7 \mathrm{~m}^{3}$ (bzw. 15 und $86 \%$ des Gesamtverbrauches) an Regenwasser für Bewässerungszwecke bereitgestellt werden. Ausgehend von der Wetter- 

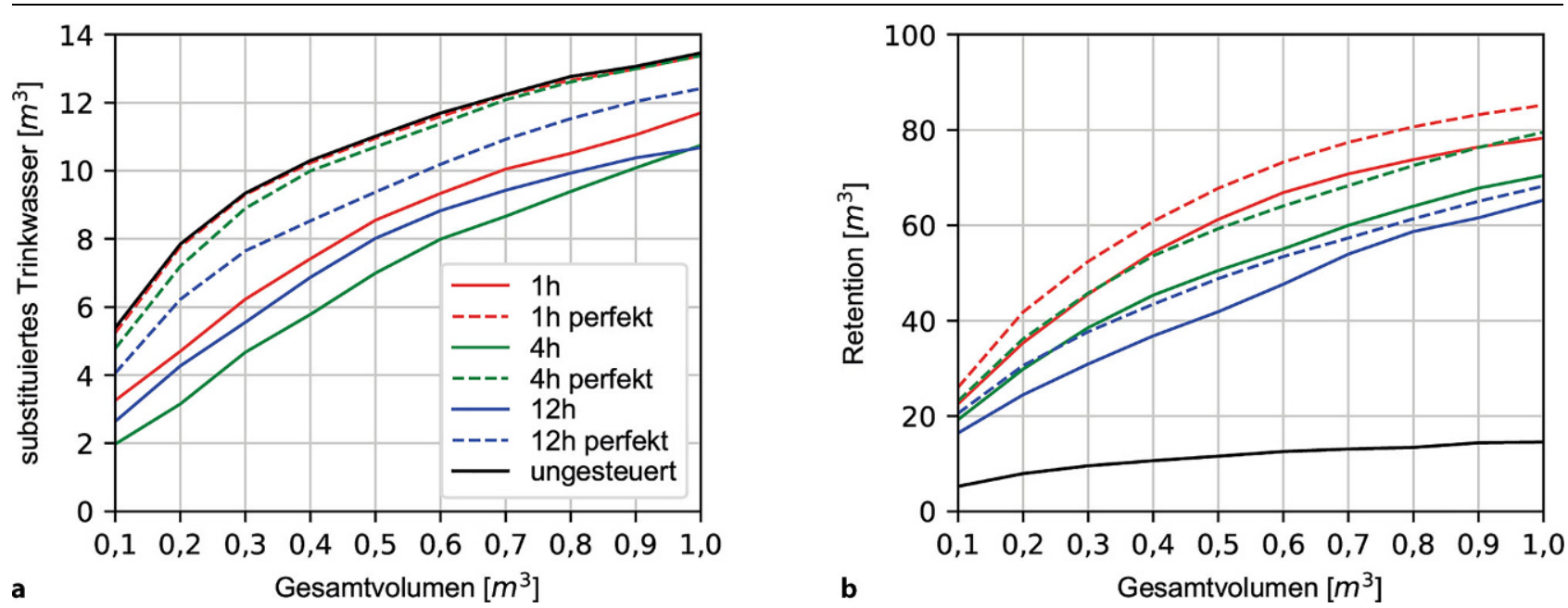

Abb. 6 Simulationsergebnisse für verschiedene Volumen sowie Akkumlationszeiten der Wettervorhersage für zwei Perfomanceindikatioren: (a) bereitgestelltes Regenwasser für Bewässerungszwecke und (b) Gesamtvolumen an zurückgehaltenem Niederschlagswasser

vorhersage wird der Zufluss zur SRT abgeschätzt und anhand dieser Abschätzung wird die SRT entleert. Wird mehr Niederschlag prognostiziert als tatsächlich fällt, wird die SRT zu viel entleert. Dadurch ist die SRT am Ende des Niederschlagsereignisses nicht vollständig gefüllt, wodurch die Bewässerungsmenge reduziert wird. Die herkömmliche Regentonne stellt den höchsten Anteil an Regenwasser für die Bewässerung zur Verfügung, da hier eine Entnahme nur für die Bewässerung erfolgt. Dadurch ist die Regentonne am Ende des Niederschlagsereignisses maximal (jedoch nicht notwendigerweise vollständig) gefüllt. Der Vergleich mit der perfekten Wettervorhersage zeigt, dass bei kurzen Akkumulationszeiten (4h) die Ergebnisse jenen der herkömmlichen Regentonne ähnlich sind. Für längere Akkumulationszeiten (12h) kann deutlich weniger Regenwasser für die Bewässerung bereitgestellt werden. Unter Berücksichtigung eines längeren Zeitraumes wird die SRT deutlich vor dem Eintritt des Niederschlagsereignisses entleert. Wird beispielsweise ein Niederschlag für 6:00 Uhr prognostiziert, beginnt sich die SRT bereits um 18:00 Uhr des Vortages $\mathrm{zu}$ entleeren. Dadurch ist zum Zeitpunkt der Bewässerung (23:00 Uhr) die SRT bereits teilweise entleert, wodurch weniger Regenwasser für Bewässerungszwecke zur Verfügung steht.

\subsubsection{Retention Regenwasser}

Für das betrachtete Sommerhalbjahr 2015 betrug der Gesamtabfluss von der $165 \mathrm{~m}^{2}$ großen Dachfläche des Einfamilienhauses $107 \mathrm{~m}^{3}$. Wie in Abb. 6b ersichtlich, beträgt die Retentionsmenge für die ungesteuerte Regentonne, je nach Regentonnengröße, zwischen 5 und $14,5 \mathrm{~m}^{3}$. Im Vergleich dazu erhöht sich durch das Hinzufügen der zukünftigen Wetterentwicklung und Steuerung der SRT das Gesamtretentionsvolumen deutlich. Bereits für das kleinste Speichervolumen mit $0,1 \mathrm{~m}^{3}$ und eine Akkumulationszeit von $4 \mathrm{~h}$ beträgt das Gesamtretentionsvolumen $19,4 \mathrm{~m}^{3}$ und ist damit deutlich höher als bei der ungesteuerten Regentonne mit einem Gesamtretentionsvolumen von $5 \mathrm{~m}^{3}$. Insgesamt konnte je nach Volumen der SRT zwischen 16,4 und 77,8 $\mathrm{m}^{3}$ an Niederschlag temporär zurückgehalten werden. Auch ist ein Unterschied zwischen den verschiedenen Akkumulationszeiten erkennbar. Bei kurzen Akkumulationszeiten ist die Qualität der Wettervorhersage höher, wobei bei einer Akkumulationszeit von $1 \mathrm{~h}$ am meisten Niederschlag zurückgehalten werden kann. Mit zunehmender Akkumulationszeit nimmt das Gesamtretentionsvolumen ab. Letztendlich sind die Abweichungen zwischen einer perfekten Wettervorhersage (als theoretisches Maximum des Gesamtretentionsvolumens) und der realen Wettervorhersage bei kleinen Gesamtvolumen (ca. 25\% Differenz bei einer SRT mit $0,1 \mathrm{~m}^{3} \mathrm{Ge}$ samtvolumen) höher als bei SRT mit größeren Gesamtvolumen (ca. 10\% Differenz).

Dadurch, dass die Einsparung der Trinkwassermenge durch das Hinzufügen der Wettervorhersage in die Kontrollstrategie abnimmt, besteht eine der Hauptherausforderungen für eine smarte, multifunktionale Regenwasserbewirtschaftung in der richtigen Vorhersage der zukünftigen Niederschlagsmengen. In Abhängigkeit von der Wetterprognose wird der Zufluss abgeschätzt und die SRT, falls erforderlich, entleert. Da die Vorhersagefehler mit größeren Zeitschritten der Wettervorhersage zunehmen, ist eine vereinfachte Abschätzung ohne Berücksichtigung des Vorhersagefehlers nicht geeignet, um im Idealfall eine vollgefüllte SRT am Ende des Niederschlagsereignisses zu gewährleisten. Die Berücksichtigung des Vorhersagefehlers ist besonders bei kleinen Speichereinheiten mit einer hohen Frequenz an Entleerungs- und Füllungsvorgängen wichtig.

In zukünftigen Untersuchungen sollte die Wirksamkeit der SRT auf die Reduktion des Spitzenabflusses im Kanalnetz eines urbanen Gebiets sowie Einfluss auf Mischwasserentlastungen berücksichtigt werden. Eine integrative Betrachtung einer Vielzahl von SRT und des Kanalnetzes ist erforderlich, um den Spitzenabfluss reduzieren zu können. Beispielsweise können die SRT ohne eine gezielte Steuerung bereits vor dem Spitzenabfluss vollständig gefüllt sein. Zusätzlich ist bei einer großflächigen Implementierung der SRT eine abgestimmte Kontrollstrategie bezüglich 
des Öffnens und Schließens notwendig. Im Extremfall kann beispielsweise das gleichzeitige Entleeren von mehreren SRT für die Bereitstellung von Retentionsvolumen zu einer Überlastung des Kanalsystems führen und muss daher koordiniert werden.

\section{Zusammenfassung und Schlussfolgerung}

Regentonnen sind dezentrale Speichereinheiten, die für eine fortgeschrittene Regenwasserbewirtschaftung genutzt werden können. Bei Niederschlag können sie zusätzliches Retentionsvolumen zur Verfügung stellen und bei Trockenwetter bieten sie das gespeicherte Regenwasser als Alternative zu Trinkwasser für Bewässerungszwecke an. Vorteile solcher Regentonnen sind die einfache Implementierung durch den geringen Platzbedarf und die Möglichkeit, sie nachträglich in existierende Systeme zu integrieren.

In der vorliegenden Arbeit wurde das Konzept der smarten Regentonnen (SRT) vorgestellt. SRT sind kleine Speichervolumen $\left(<1,0 \mathrm{~m}^{3}\right)$, die in Echtzeit kontrolliert werden können. Im Vergleich zum zentralen Gesamtsystem (Kanalsystem) stellen einzelne Anlagen zwar einen kleinen Speicher dar, jedoch ergeben sich durch einen flächendeckenden Einsatz relevante Volumen und durch neue Möglichkeiten im Betrieb solcher Anlagen, wie zum Beispiel die Einbindung in eine smarte Wasserinfrastruktur oder der Wettervorhersage in die Kontrollstrategie, können SRT effizient eingesetzt werden. Einerseits können Spitzenabflüsse im Kanalsystem sowie die Häufigkeit von Misch- wasserentlastungen reduziert werden, andererseits kann der Trinkwasserverbrauch durch die Nutzung von Regenwasser zur Bewässerung vermindert werden. Es wurden numerische Simulationen auf Haushaltsebene für den Wirksamkeitsnachweis durchgeführt, um die Auswirkungen der SRT auf die Wasserversorgung sowie auf die Siedlungsentwässerung $\mathrm{zu}$ demonstrieren. Während hier die Gesamtretentionsmenge ermittelt wurde, wurden die Auswirkungen auf den Spitzenabfluss nicht näher betrachtet. Für die Simulationen wurden Niederschlagsdaten in 1-min-Intervallen, Temperaturdaten in 10-min-Intervallen sowie historische Wettervorhersagen in 15-min-Schritten aus dem Jahr 2015 verwendet. Dabei konnte festgestellt werden, dass in Abhängigkeit vom Volumen der SRT und der Akkumulationszeit zwischen 15 und $86 \%$ an Trinkwasser durch die Verwendung von Regenwasser für Bewässerungszwecke eingespart werden kann. Zudem konnten insgesamt zwischen 16,4 und $77,8 \mathrm{~m}^{3}$ an Niederschlag temporär zurückgehalten werden. Durch das Hinzufügen der Wettervorhersage in die Kontrollstrategie konnte das Retentionsvolumen auch für kleine Speichervolumen $\left(<0,2 \mathrm{~m}^{3}\right)$ gegenüber einer ungesteuerten Regentonne deutlich erhöht werden. Jedoch wurde durch die Einbindung der zukünftigen Wetterentwicklung die Effektivität zur Einsparung an Trinkwasser leicht verringert.

Werden die SRT großflächig in einem Siedlungsgebiet implementiert, steht durch die große Anzahl ein relevantes zusätzliches Retentionsvolumen zur Verfügung. Werden beispielsweise
66 Einfamilienhäuser (reduzierte Gesamtfläche entspricht $1 \mathrm{ha}$ ) mit einer SRT und jeweils einem Volumen von 2501 ausgestattet, können $16,5 \mathrm{~m}^{3}$ an zusätzlichem Retentionsvolumen ohne hohen Bauaufwand bereitgestellt werden. Jedoch sind neue Anreize (zum Beispiel Fördermodelle, Bewilligungsverfahren für zentrale Systeme) für die Bevölkerung sowie auch Betreiber notwendig, damit diese Maßnahmen unterstützt und in zukünftigen Forschungstätigkeiten weiter thematisiert werden.

Förderung Diese Publikation entstand im Rahmen des Projekts „Smart Water City“. Dieses Projekt wird aus den Mitteln des Klima- und Energiefonds gefördert und im Rahmen des Programms „Smart Cities Demo-Living Urban Innovation 2018“ (Projekt 872123) durchgeführt.

Funding Open access funding provided by University of Innsbruck and Medical University of Innsbruck.

Open Access Dieser Artikel wird unter der Creative Commons Namensnennung 4.0 International Lizenz (http:// creativecommons.org/licenses/by/4. $0 /$ deed.de) veröffentlicht, welche die Nutzung, Vervielfältigung, Bearbeitung, Verbreitung und Wiedergabe in jeglichem Medium und Format erlaubt, sofern $\mathrm{Sie}$ den/die ursprünglichen $\mathrm{Au}$ tor(en) und die Quelle ordnungsgemäß nennen, einen Link zur Creative Commons Lizenz beifügen und angeben, ob Änderungen vorgenommen wurden.

\section{Literatur}

A review Environmental Modelling \& Software 72:198-214 https://doi.org/10.1016/j.envsoft. 2015.07.012

DWA-M 153 (2007): Handlungsempfehlungen zum Umgang mit Regenwasser

EN 16941-1 (2018): EN 16941-1 On-site non-potable water systems. CEN,

Fewkes, A., Butler, D. (2000): Simulating the performance of rainwater collection and reuse systems using behavioural models Building Services Engineering Research and Technology 21:99-106 https://doi.org/10.1177/ 014362440002100204

Fletcher, T.D. et al. (2015): SUDS, LID, BMPs, WSUD and more-The evolution and application of terminology surrounding urban drainage Urban Water Journal 12:525-542 https://doi.org/ 10.1080/1573062x.2014.916314

Fletcher, T.D., Andrieu, H., Hamel, P. (2013): Understanding, management and modelling of urban hydrology and its consequences for receiving waters: A state of the art Advances in Water
Resources 51:261-279 https://doi.org/10.1016/j. advwatres.2012.09.001

García, L., Barreiro-Gomez, J., Escobar, E. Téllez, D., Quijano, N., Ocampo-Martinez, C. (2015): Modeling and real-time control of urban drainage systems: A review Advances in Water Resources 85:120-132 https://doi.org/10.1016/j. advwatres.2015.08.007

Haiden, T., Kann, A., Wittmann, C., Pistotnik, G., Bica, B., Gruber, C. (2011): The Integrated Nowcasting through Comprehensive Analysis (INCA) System and Its Validation over the Eastern Alpine Region Weather and Forecasting 26:166-183 https://doi.org/10.1175/ 2010waf2222451.1

Huang, C.-L., Hsu, N.-S., Wie, C.-C., Luo, W.J. (2015): Optimal Spatial Design of Capacity and Quantity of Rainwater Harvesting Systems for Urban Flood Mitigation Water 7:5173-5202 https://doi.org/10.3390/w7095173

Kerkez, B. et al. (2016): Smarter Stormwater Systems Environmental Science \& Technology 
50:7267-7273 https://doi.org/10.1021/acs.est. $5 \mathrm{~b} 05870$

Kleidorfer, M., Moderl, M., Fach, S., Rauch, W. (2009): Optimization of measurement campaigns for calibration of a conceptual sewer model Water Science \& Technology 59:1523-1530 https://doi.org/10.2166/wst.2009.154

Di Matteo, M., Liang, R., Maier, H.R., Thyer, M.A., Simpson, A.R., Dandy, G.C., Ernst, B. (2019): Controlling rainwater storage as a system: An opportunity to reduce urban flood peaks for rare, long duration storms Environmental Modelling \& Software 111:34-41 https://doi.org/ 10.1016/j.envsoft.2018.09.020

Mekki, K., Bajic, E., Chaxel, F., Meyer, F. (2018): A comparative study of LPWAN technologies for large-scale IoT deployment ICT Express https:// doi.org/10.1016/j.icte.2017.12.005

Neunteufel, R., Richard, L., Perfler, R., Tuschel, S., Böhm, K., Haas, E. (2012): Wasserverbrauch und Wasserbedarf - Auswertung empirischer Daten zum Wasserverbrauch. Bundesministerium für Land- und Forstwirtschaft, Umwelt und Wasserwirtschaft,

Notaro, V., Liuzzo, L., Freni, G. (2017): Evaluation of the optimal size of a rainwater harvesting system in Sicily Journal of Hydroinformatics 19:853-864 https://doi.org/10.2166/hydro.2017. 150

Oberascher, M., Zischg, J., Palermo, S.A., Kinzel, C., Rauch, W., Sitzenfrei, R. (2019): Smart
Rain Barrels: Advanced LID Management Through Measurement and Control. In: Cham (2019): New Trends in Urban Drainage Modelling. UDM 2018. Green Energy and Technology. Springer, Cham pp 777-782

ÖWAV-Regelblatt 19 (2007): Richtlinien für die Bemessung von Mischwasserentlastungen.

Prince George's County (1991): Low-Impact Develompent Design Strategies: An Integrated Design Approach. Prince George's County Department of Environmental Resources, Maryland,

Rossman, L.A. (2015): Storm Water Management Model User's Manual Version 5.

Sample, D.J., Liu, J. (2014): Optimizing rainwater harvesting systems for the dual purposes of water supply and runoff capture Journal of Cleaner Production 75:174-194 https://doi.org/ 10.1016/j.jclepro.2014.03.075

Sitzenfrei, R., Rauch, W. (2014): Integrated hydraulic modelling of water supply and urban drainage networks for assessment of decentralized options Water Science and Technology 70:1817-1824 https://doi.org/10.2166/wst.2014 326

Sitzenfrei, R. (2015): An integrated view of the urban water cycle: A shift in the paradigm? Priv.Doz. habilitation-thesis (postdoctoral qualification), University of Innsbruck

Statistik Austria (2018a): Fertiggestellte Wohnungen und Gebäude 2005 bis 2017. https:// www.statistik.at/web_de/statistiken/menschen und gesellschaft/wohnen/wohnungs und gebaeudeerrichtung/fertigstellungen/index. html. Accessed 01.032018

Statistik Austria (2018b): Privathaushalte nach Haushaltsgröße, Bundesländern und Alter der Haushaltsreferenzperson - Jahresdurchschnitt 2017. https://www.statistik.at/web_de/ statistiken/menschen_und_gesellschaft/ bevoelkerung/haushalte_familien_

lebensformen/haushalte/023302.html. Accessed 01.032018

Ward, S., Memon, F.A., Butler, D. (2012): Performance of a large building rainwater harvesting system Water Res 46:5127-5134 https://doi.org/ 10.1016/j.watres.2012.06.043

Yazdanfar, Z., Sharma, A. (2015): Urban drainage system planning and design-challenges with climate change and urbanization: a review Water Science and Technology 72:165-179 https:// doi. org/10.2166/wst.2015.207

Hinweis des Verlags Der Verlag bleibt in Hinblick auf geografische Zuordnungen und Gebietsbezeichnungen in veröffentlichten Karten und Institutsadressen neutral. 